\title{
ADOÇÃO E USO DA AGRICULTURA DE PRECISÃO NA REGIÃO DAS MISSÕES DO RIO GRANDE DO SUL
}

\author{
R. C. ANTONINI ${ }^{1}$, R. P. BORTOLOTTO ${ }^{2}$, J. F. ZAMBERLAN ${ }^{3}$, D. DALLA NORA ${ }^{4}$, M. P. B. PASINI ${ }^{5}$, J. E. FIORIN ${ }^{6}$
${ }^{1}$ Agrofel, ${ }^{2,3,5,6}{ }^{\text {Universidade de Cruz Alta, }{ }^{4} \text { Instituto Federal Farroupilha }}$
rafaelpbortolotto@unicruz.edu.br ${ }^{2}$ \\ Submetido 15/09/2017 - Aceito 11/06/2018 \\ DOI: $10.15628 /$ holos.2018.6297
}

\section{RESUMO}

Embora sendo cada vez mais adotada no Brasil, existem poucos estudos sobre o processo de adoção e uso de tecnologias de AP no país. O objetivo deste estudo foi avaliar a percepção, grau de adoção e perfil dos produtores rurais quanto à utilização da técnica de AP na Região Noroeste do RS. O trabalho caracterizou-se como uma pesquisa exploratória quanto a sua finalidade. $O$ público-alvo da entrevista foram produtores rurais assistidos pela Cooperativa Tritícola Regional São-Luizense Ltda. Para a obtenção das descrições experienciais de cada produtor foram utilizados questionários semiestruturados, sendo um com perguntas abertas e fechadas e outro utilizando-se a Escala Likert. Os dados foram analisados calculando-se a frequência das respostas dadas e análise de discurso, e a associação entre as variáveis e os produtores investigados foi identificada mediante o uso de análise estatística multivariada. A AP ainda é recente na Região Noroeste do RS, sendo utilizadas poucas ferramentas, restringindo-se à aplicação à taxa variável de insumos, muito centrada na produção de soja, principalmente. É utilizada por produtores de diferentes níveis de escolaridade, idade e tamanho de área.

PALAVRAS-CHAVE: Taxa Variável, soja, agricultura, escolaridade, adoção.

\section{ADOPTION AND USE OF PRECISION AGRICULTURE IN THE REGION OF MISSIONS - RIO GRANDE DO SUL}

\begin{abstract}
Despite being increasingly adopted in Brazil, there are few studies on the process of adoption and use of PA technologies in the country. The aim of this study was to evaluate the perception degree of adoption and profile of farmers regarding the use of the AP technique in the area of Missions -RS. The study was characterized as an exploratory research as its purpose. The target audience of the interview was farmers by Cooperative Tritícola Regional São-Luizense. To obtain experiential descriptions of each producer, semi-structured questionnaires were used, one with open and closed
\end{abstract}

questions and others using Likert Scale. Data were analyzed by calculating the frequency of answers, discourse analysis and the association between the variables and investigated producers was identified through the use of multivariate statistical analysis. AP is still fresh in the Region and RS Northwest. Few tool is still used, restricting the application of the variable rate focused mainly in the production of soybean mainly. Used by producers of different education levels, age and area size.

KEYWORDS: Variable rate, soy, agriculture, levels of education, adoption. 


\section{INTRODUÇÃO}

A agricultura tem passado por diversas transformações, exigindo do produtor maior nível de especialização, capacidade de gerenciamento e profissionalismo. Os produtores, além de administradores, devem estar diretamente ligados na coleta de informações a campo, interagindo com novas técnicas e tomando decisões eficazes de manejo. Para que isso possa acontecer, é crucial a obtenção de informações sobre os fatores de produção que interagem na lavoura e sobre como eles podem ser maximizados.

O Rio Grande do Sul (RS), em especial no noroeste do Estado, ocupa posição de destaque no agronegócio nacional e mundial, posição conquistada com o auxílio de pesquisas, melhoramento genético de plantas, aprimoramento da mecanização, adoção de técnicas de manejo adequado do solo, em especial do sistema plantio direto e, associado a este, a agricultura de precisão (AP).

A AP está inserida em um cenário de demandas como um conjunto de ferramentas que permite aprimorar a forma de se fazer a agricultura, diminuindo incertezas na hora da tomada de decisão. O RS se destaca na produção nacional de grãos como soja, milho, trigo, arroz (irrigado) e aveia (grão e pastagem), além da fabricação de implementos agrícolas na área da agricultura de precisão, em especial nos municípios de Horizontina, Não-Me-Toque, Santa Rosa e Canoas. Devido à importância brasileira no agronegócio mundial e ao destaque nacional do RS entre os principais produtores de grãos e como Estado em que se pratica a AP, fazer uma análise dos motivos da adoção desta tecnologia pelos produtores rurais no RS, em especial nas Missões, constitui-se um estudo pertinente de grande relevância.

O crescimento da AP foi alavancado por avanços tecnológicos envolvendo a liberação do uso de satélites para posicionamento global (SPG ou GPS) para uso civil. Com isso, a agricultura foi beneficiada através do uso de ferramentas como aplicação de insumos em taxas variáveis, através do estudo da variabilidade espacial, possibilitando manejar a área agrícola de acordo com os fatores edafoclimáticos, não mais o fazendo de forma homogênea.

A AP (também denominada "Precision Farming", "Precision Agriculture" e "Site Specific Crop Management") é um sistema de gerenciamento que investiga a variabilidade espacial. A AP é um conjunto de tecnologias destinadas ao manejo de solos, culturas e insumos, que objetiva melhorar o gerenciamento do sistema de produção agrícola em todas as etapas, desde a semeadura até a colheita, sendo ferramenta de gestão do sistema produtivo agrícola, considerando a variabilidade espacial e temporal, visando minimizar efeito negativo ao meio ambiente e maximizar retorno econômico (Inamasu et al. 2011). Segundo Molin (2002), uma definição mais atual de AP com visão sistêmica do conjunto de ações que a compõe pode ser adotada: a AP seria, acima de tudo, um sistema de gestão ou de gerenciamento da produção agrícola que emprega um conjunto de tecnologias e procedimentos para que as lavouras e sistemas de produção sejam otimizados, tendo como elemento-chave o manejo da variabilidade da produção e dos fatores envolvidos.

A necessidade de atender a demanda por alimentos não pode deixar que nos descuidemos dos aspectos relacionados ao meio ambiente, e ações antrópicas podem levar ao esgotamento dos recursos naturais, em especial solo e água. A adoção de tecnologias que 
busquem a sustentabilidade da atividade agrícola é bem-vinda. Neste aspecto, a AP busca extrair o máximo de produtividade sem agressão do meio, ao mesmo tempo em que reduz o risco de impacto ambiental (Hong et al., 2007; Bragagnolo et al., 2013).

Portanto, a AP não está ligada totalmente a investimentos tecnológicos e implementos agrícolas, e sim a um manejo completo da área agricultável, visando a um aumento constante de produção e aproveitamento otimizado dos recursos naturais, gerando ao produtor rural um maior rendimento em sua produção e, consequentemente, produzindo mais alimentos para suprir as demandas mundiais.

Embora sendo cada vez mais adotada no Brasil, existem poucos estudos sobre o processo e uso de tecnologias de AP no país. O objetivo deste estudo foi avaliar a percepção, grau de adoção e perfil dos produtores rurais quanto à utilização da técnica de AP na Região das Missões do RS.

\section{FUNDAMENTAÇÃO TEÓRICA}

A inovação tecnológica é elemento chave para a busca de novas oportunidades de mercado. Na agricultura, ela constitui-se de máquinas, equipamentos, defensivos agrícolas, fertilizantes, uso da biotecnologia, além da utilização de ferramentas da AP (Cirani \& Moraes, 2010). A AP apresenta-se como conjunto de ferramentas capaz de auxiliar o produtor rural a identificar as estratégias a serem adotadas para aumentar a eficiência no gerenciamento da agricultura (Carvalho et al., 2009). Ela tem sido amplamente utilizada no mundo para incrementar a produção, melhorar o retorno econômico e reduzir os impactos ambientais (Blackmore et al., 2003), principalmente para culturas de grãos (Bramley, 2009), contribuindo para sustentabilidade do sistema (Bongiovanni \& Lowenberg-Deboer, 2004), porém, no início, de forma mais lenta que o previsto (McBratney et al., 2005).

Antes mesmo da Revolução Industrial e do processo de mecanização da atividade agrícola, os agricultores já se mostravam capazes de reconhecer a variabilidade espacial de certas características físico-químicas e biológicas das áreas cultivadas. A própria divisão dessas áreas em talhões reflete essa capacidade de discernimento. Até então, o uso de trabalho braçal e/ou tração animal permitia aos agricultores tratar áreas com menor ou maior fertilidade ou com infestação de pragas, doenças e plantas daninhas de forma diferenciada (Fraisse, 1998). 0 foco da AP é o gerenciamento da variabilidade espacial da produção e dos fatores nela envolvidos, realizado por meio de tecnologias recentes, adaptadas para o meio agrícola, com o objetivo de possibilitar a redução do uso de insumos e do impacto sobre o meio ambiente (Ciranl et al., 2010; Ciranl \& Moraes, 2010).

Os fundamentos da AP moderna, segundo a literatura, surgiram em 1929, nos Estados Unidos da América, e foram descritos por Linsley e Bauer na circular $n^{\circ} 346$ da Estação Experimental Agrícola da Universidade de Illinois (Goering, 1993). Nessa época, havia se constatado a existência de grandes variações quanto à necessidade de calagem em determinada área e que a aplicação de calcário deveria respeitar essa variabilidade.

No Brasil, as primeiras ações de pesquisa na área foram realizadas na Escola Superior de Agricultura "Luiz de Queiroz", da Universidade de São Paulo (ESALQ-USP) em 1997, onde um trabalho pioneiro com a cultura de milho resultou no primeiro mapa de variabilidade de 
colheita do Brasil (Balastreire et al., 1997). Atualmente, a AP é uma tecnologia que está sendo aplicada em diferentes culturas no Brasil, que são importantes para garantir a posição do País na produção agrícola. (Silva et al., 2011).

Nos primeiros dez anos, as pesquisas na área concentravam-se no desenvolvimento de sensores. Com a disponibilização do GPS - conjunto de satélites americanos que foram empregados na "Guerra Fria" - a partir de 1990, houve considerável incremento nas pesquisas voltadas para a AP. Em geral, todos os grandes centros de pesquisa em engenharia agrícola e agricultura estão trabalhando no desenvolvimento de técnicas de $\mathrm{AP}$, e as indústrias de máquinas agrícolas têm acompanhado este desenvolvimento. Atualmente, grande número de indústrias fabricam máquinas e sistemas de controle, e há empresas especializadas no desenvolvimento de "softwares" para a AP (Mantovani \& Gomide, 2000). A AP faz uso intenso de sistemas de posicionamento por satélite e de sistemas de informações geográficas (GIS9), permitindo o tratamento e análise de dados coletados no campo. A análise dos dados permite a otimização do uso de insumos agrícolas, possibilitando ganhos econômicos para o agricultor e reduzindo o impacto ambiental da atividade (Gimenez \& Molin, 2004).

É possível identificar áreas manejadas dessa forma em numerosos locais do Brasil. Por exemplo, nas regiões coloniais do Rio Grande do Sul, com propriedades de topografia acidentada e com pequenas áreas de cultivo, é possível observar uma agricultura diversificada, em que a alocação das culturas é feita de acordo com as características de cada talhão e o produtor conhece o potencial de cada área e pratica o manejo específico em cada situação. Esse tipo de manejo, que prescinde de equipamentos de alta tecnologia, pode ser considerado também uma forma, embora empírica e muitas vezes inconsciente, de agregar maior precisão à atividade agrícola. Assim, ao contrário do que muitos pensam, os princípios da AP não são uma novidade nas áreas cultivadas intensivamente e nas quais o trabalho era realizado manualmente (Molin, 2002).

A AP, na atualidade, ganha uma definição mais sistêmica, podendo ser conceituada como uma nova forma de gestão ou de gerenciamento da produção agrícola, e não apenas como um conjunto de ferramentas para o tratamento localizado da lavoura (CIRANI et al., 2010). A AP surge como uma importante ferramenta para maximizar o gerenciamento da atividade agrícola de elevado custo de produção (Ferraz et al., 2011).

Atualmente, no Brasil e no RS, existem pesquisas relacionadas com AP no que diz respeito à compactação do solo (Girardello et al., 2014a), tráfego controlado (Girardello et al., 2014b), manejo de pastagens e culturas de grãos (SILVA et al., 2014), sensores de adubação nitrogenada (Bragagnolo et al., 2013), zonas de manejo, fertilidade do solo (Santi et al., 2013; Demattê et al., 2014), entre outras, sendo consideradas bem desenvolvidas as pesquisas que envolvem solo e seus manejos, porém são poucos os estudos na área de defesa fitossanitária. Dessa forma, as ferramentas da AP devem ser consideradas como um importante auxílio no manejo de lavouras (Ferraz et al., 2011). A grande maioria dos esforços das pesquisas têm se concentrado nos aspectos técnicos da aplicação das ferramentas de AP e pouco tem sido feito para entender a adoção e os condicionantes de tal atitude (Anselmi, 2012). 


\section{MÉTODO}

A referida seção aborda os procedimentos metodológicos que foram utilizados a fim de atingir o objetivo proposto. A seguir, são descritos os principais procedimentos utilizados nesta pesquisa.

O trabalho caracterizou-se como uma pesquisa exploratória quanto a sua finalidade, que segundo Hair et al. (2005) é utilizada quando se tem poucas informações acerca do objeto de pesquisa. Vergara (2011) diz que a pesquisa exploratória se destina a quando se sabe pouco ou quase nada sobre um determinado fenômeno ou objeto, possuindo uma natureza de sondagem.

Foi realizada uma pesquisa de abordagem quanti-qualitativa. O público-alvo da entrevista foram produtores rurais assistidos pela Cooperativa Tritícola Regional São-Luizense Ltda. Sua escolha foi devido à importância da região no setor agropecuário. Para manter o anonimato das referidas organizações, iremos nos referir a elas por meio de letras (Tabela 1). Segundo Gil (2010), para a realização desse tipo de entrevista se faz necessário um clima de receptividade, sendo importante assegurar ao entrevistado a confidencialidade dos dados por ele fornecidos. Também se faz necessário expor o tempo para a coleta dos dados e um local adequado e tranquilo para realização da entrevista. No total, foram entrevistados 30 produtores rurais da Região das Missões, assistidos pela Coopatrigo.

Para a obtenção das descrições experienciais de cada produtor foram utilizados questionários semiestruturados, sendo um com perguntas abertas e fechadas e outro utilizando-se a Escala Likert com cinco níveis, em que 1 equivale a discordo totalmente e 5 equivale a concordo totalmente. Segundo Teixeira (2005), o respondente indica o seu grau de concordância ou discordância para cada um dos itens em lugar de diferenciar os itens entre os quais concorda e aqueles com os quais discorda. Um item Likert é apenas uma afirmação a qual o sujeito pesquisado responde através de um critério que pode ser objetivo ou subjetivo. Normalmente, o que se deseja medir é o nível de concordância ou não concordância à afirmação. A Escala Likert, desenvolvida por Rensis Likert, é a variação mais frequentemente usada da escala de classificação somatória, a qual consiste em afirmações que expressam atitudes favoráveis ou desfavoráveis em relação ao objeto de interesse (Cooper \& Schindler, 2011).

Os dados referentes à Escala Likert foram analisados calculando-se a frequência das respostas dadas. No que tange ao questionário semiestruturado, as respostas das perguntas abertas foram analisadas mediante análise de discurso. As perguntas fechadas sofrerão tratamento estatístico de frequência. A associação entre as variáveis e os produtores investigados foi identificada mediante o uso de análise estatística multivariada - Análise Fatorial/Análise de Componente Principal (AF/ACP). Para a análise dos dados utilizou-se o Software SPSS (Statistical Package Social Science) versão 20.0. A AF/ACP demanda três etapas: a primeira consiste no preparo da matriz de correlação [R]; a segunda na extração dos fatores comuns e da possível redução de variáveis explicativas e, por último, na rotação dos eixos relativos aos fatores comuns, simplificando a solução (Toledo \& Nicolella, 2002). 
Tabela 1: Codificação dos participantes da entrevista

\begin{tabular}{c|cc}
\hline PRODUTOR & ESCOLARIEDADE & TEMPO DE USO AP \\
\hline R.1 & Superior incompleto & 1 ano \\
R.2 & Superior incompleto & 3 anos \\
R.3 & Superior completo & 1 ano \\
R.4 & Ensino médio incompleto & 2 anos \\
R.5 & Ensino médio incompleto & 2 anos \\
R.6 & Ensino médio completo & 2 anos \\
R.7 & Ensino médio completo & 2 anos \\
R.8 & Superior completo & 2 anos \\
R.9 & Ensino médio completo & 2 anos \\
R.10 & Ensino médio incompleto & 1 ano \\
R.11 & Ensino médio incompleto & 3 anos \\
R.12 & Superior completo & 2 anos \\
R.13 & Superior completo & 3 anos \\
R.14 & Ensino médio completo & 2 anos \\
R.15 & Ensino médio completo & 2 anos \\
R.16 & Ensino médio completo & 3 anos \\
R.17 & Superior completo & 2 anos \\
R.18 & Ensino médio completo & 3 anos \\
R.19 & Ensino médio incompleto & 1 ano \\
R.20 & Ensino médio completo & 2 anos \\
R.21 & Ensino médio completo & 2 anos \\
R.22 & Ensino médio completo & 3 anos \\
R.23 & Ensino médio incompleto & 1 ano \\
R.24 & Superior completo & 2 anos \\
R.25 & Ensino médio completo & 3 anos \\
R.26 & Ensino médio completo & 2 anos \\
R.27 & Superior completo & 2 anos \\
R.28 & Ensino médio completo & 3 anos \\
R.29 & Superior completo & 1 anos \\
\hline &
\end{tabular}

\section{RESULTADOS E DISCUSSÕES}

A análise fatorial (AF) promoveu redução no número de variáveis, foram retiradas as variáveis "uso de AP em terras arrendadas", "cultivos com uso de AP", "conhecimento dos procedimentos para realização da AP", "ferramentas da AP utilizadas" e "AP no manejo de insetos-pragas, doenças e plantas daninhas", uma vez que o melhor ajuste ocorreu com a inclusão das demais variáveis analisadas (Tabela 2). O modelo que melhor se ajustou aos dados obtidos pelo questionário foi composto por três fatores, explicando, cerca de $75 \%$ da variância total das variáveis originais. 
Tabela 2: Matriz de cargas fatoriais após rotação ortogonal pelo Método Varimax para os dados do questionário

\begin{tabular}{ccccc}
\hline & \multicolumn{4}{c}{ Fatores } \\
\cline { 2 - 5 } Quantos hectares cultiva & 1 & 2 & 3 & Comunalidades \\
\cline { 2 - 5 } Hectares com AP & 0,982 & 0,042 & 0,089 & 0,949 \\
Terras próprias & 0,881 & $-0,062$ & 0,226 & 0,832 \\
Terras Arrendadas & 0,973 & 0,033 & 0,103 & 0,937 \\
Idade & 0,924 & 0,070 & 0,076 & 0,840 \\
Escolaridade & 0,032 & 0,750 & 0,328 & 0,566 \\
Tempo de uso da AP & 0,225 & 0,746 & $-0,115$ & 0,597 \\
É dificil aprender a usar as & 0,259 & 0,067 & 0,917 & 0,233 \\
ferramentas da AP? & $-0,087$ & $-0,534$ & $-0,063$ & 0,295 \\
Autovalor & & & & \\
\% da Variância & 4,014 & 2,438 & 1,041 & \\
\% da Variância Acumulada & 40,141 & 24,383 & 10,408 & \\
\hline
\end{tabular}

A solução fatorial extrai os fatores na ordem de sua importância. Assim, para o resultado do questionário, o primeiro fator explica 40,1\% da variabilidade dos dados (Tabela 2). Esse fator está associado a variáveis indicativas da quantidade e modo de utilização da área de terra (Quantos hectares cultiva, Hectares com AP, Terras próprias e Terras arrendadas sem AP), sendo por isso considerado o fator de quantidade e uso da terra - GIII (Figura 1).

O Fator 1 (Tabela 2) foi o que explicou a maior porcentagem da variância, as variáveis Número de hectares que cultiva e Terras próprias são as que apresentaram maior relação, sendo, assim, as que mais influenciam o uso da AP para os produtores entrevistados (Tabela 2). Todos os produtores entrevistados cultivam pelo menos 30 ha (R.30 - Tabela 1), independente do uso de AP. Fazem AP em terras próprias porque constatam benefícios, conforme relatam alguns dos entrevistados:

Utilizando AP consegui melhorar o $\mathrm{pH}$ e a fertilidade do solo da minha propriedade que até pouco tempo era áreas de campo onde agora produzo soja (R.3).

Consigo fazer a correção do solo da minha área, pois era um dos fatores que limitavam a produtividade da minha cultura (R.6).

Consigo aumentar a produtividade desta área corrigindo-a através das necessidades verificadas na análise de solos (R.7).

Corrijo as deficiências nutricionais sem gastar adubo onde não precisa, pois, minhas terras são desparelhas (R.16).

Aumentei a produtividade desta área. Onde produzia menos notei que está produzindo mais e nas manchas onde aplicava bastante fosforo hoje estou aplicando menos (R.22). 
Com utilização da AP consigo melhorar a fertilidade do solo e otimizar o uso dos insumos tendo um melhor aproveitamento dos mesmos (R.27).

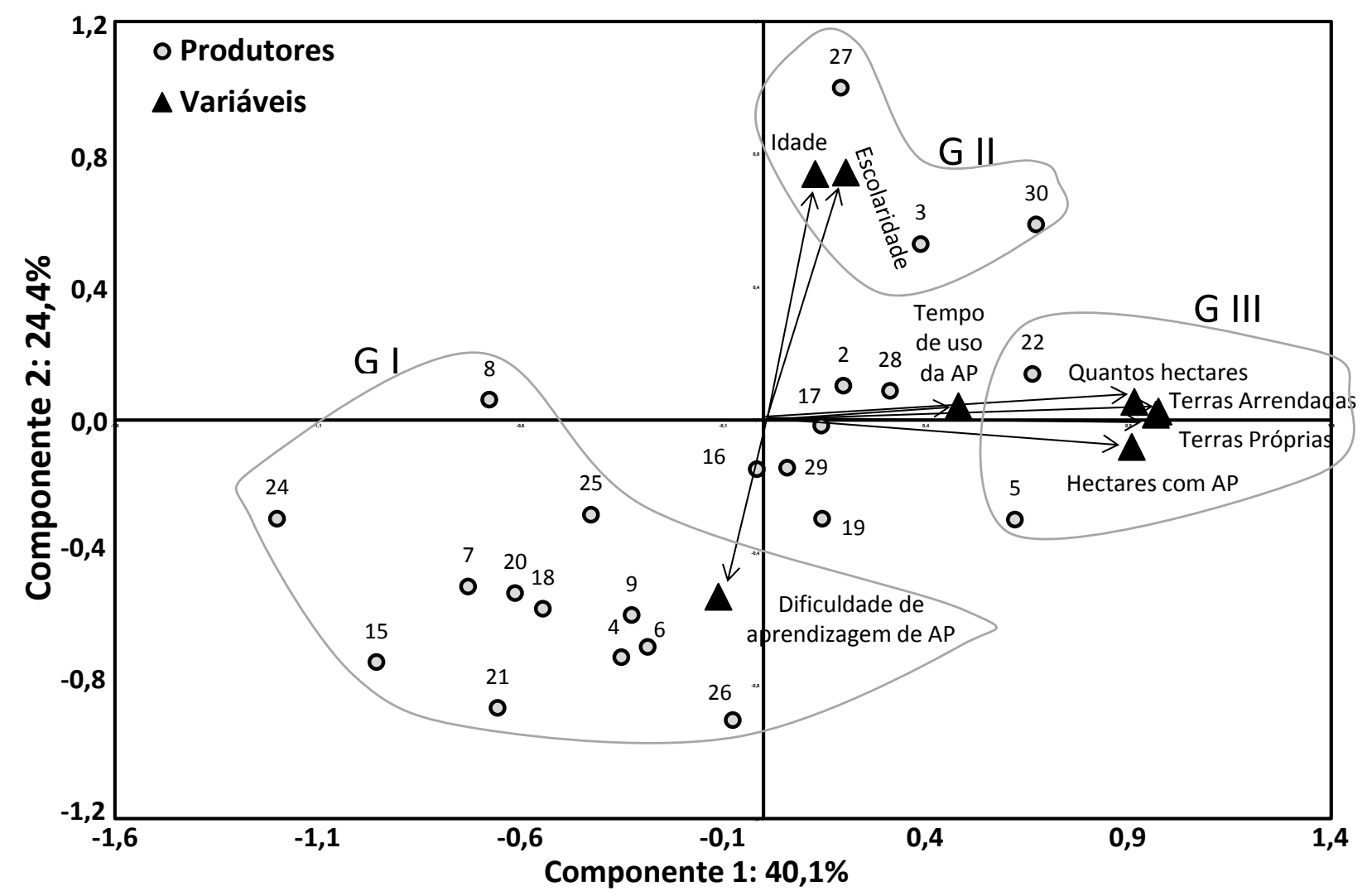

Figura 1: Biplot da associação entre as variáveis e os objetos, com destaque para as associações obtidas na análise fatorial

Outros constituintes do Fator 1 (Tabela 2), Hectares com AP e Terras arrendadas sem AP, chamam a atenção pelo fato de que todos os produtores que utilizam AP o fazem em terras próprias, não fazendo uso em terras arrendadas. Porém, o constituinte Terras arrendadas teve peso para o Fator 1, pois $57 \%$ dos produtores entrevistados arrendam terras como forma de suplementar a produção e a renda da propriedade, e também para uso de maquinários disponíveis, que assim não ficam ociosos. Além disso, podemos inferir que em pequenas propriedades há utilização de ferramentas da AP para aumentar a eficiência e a rentabilidade, pois foram registrados produtores com um mínimo de 10 ha de área com AP. Por outro lado, existem estudos que encontraram relação positiva entre tamanho da área e utilização de ferramentas da AP, ou seja, quanto maior a área cultivada, maior é o número de adotantes (Daberkow \& McBride, 2003; Adrian, 2005).

O segundo fator, que explica $24,4 \%$ da variância total dos dados originais, relaciona-se com a Experiência dos produtores (idade e escolaridade). Destaque para os produtores R.3, R.27 e R.30 (GII - Figura 1), todos possuem formação superior e idades que variam entre 31 e 38 anos, com média de 33,6 anos, o que remete a uma característica jovem e empreendedora 
na propriedade rural. Além disso, são produtores que cultivam de 20 a 30 ha de áreas com AP. Se levarmos em consideração todos os adotantes entrevistados, a média de idade foi de 43,5 anos, com variação de 28 a 58 anos, sendo que destes apenas $30 \%$ possuem formação superior. Em estudo realizado por Anselmi (2012) no Estado do Rio Grande do Sul, a idade dos adotantes de AP variou entre 19 e 63 anos, sendo que a média foi 41 anos, com mais de $44 \%$ dos entrevistados com formação superior. Os constituintes relacionados aos Fatores 1 e 2 estão ligados ao caráter inovador do uso de ferramentas da AP (Rogers, 2003; Marsh et al., 2000).

Enquanto isso, o Fator 3, com apenas 10,41\% (Tabela 2) da variância dos dados, relaciona-se com a Experiência de uso (tempo de uso da AP). Esse fator teve pouco peso, pois todos os produtores utilizam há pouco tempo a AP, variando de 1 a 3 anos, com média de utilização de 2,03 anos.

Nota-se que a variável Dificuldade de aprendizagem da AP se relaciona negativamente nos fatores, ou seja, tem pouca representatividade para os produtores, que não consideram esse fator relevante (GI - Figura 2), pois apenas $16,7 \%$ dos entrevistados consideram difícil o uso, o que pode ser demonstrado na fala de alguns dos entrevistados:

Sim. Não possuo conhecimento em informática porque até pouco tempo não tinha energia elétrica na minha propriedade e apesar de ter ido à escola passei a vida trabalhando na lavoura (R.10).

Não tenho instrução. A empresa que faz o serviço não tem contato direto comigo, apenas com quem contrato o serviço. Possuo pouca prática de informática. (R.11).

Sim, é difícil. Não tenho muito conhecimento de como funciona porque não sei informática (R.23).

Isso demonstra que uma das barreiras no uso da AP são o conhecimento e a utilização da informática. A dificuldade no uso de informática também está relacionada à faixa etária dos entrevistados, dos cinco produtores que disseram ter dificuldades ou não terem conhecimento de informática, quatro possuem idade superior a 50 anos.

A comunalidade (Tabela 2) indica o quanto da variância de cada atributo é explicado pelos fatores juntos, enquanto os autovalores indicam a importância relativa de cada fator na explicação da variância associada ao conjunto de atributos analisados. Na última coluna da Tabela 2, podem-se observar os elevados valores das comunalidades para as variáveis que compõem o fator Quantidade e uso da terra, destacando-se as variáveis Terras próprias e Terras arrendadas, o que demonstra forte relação com os fatores retidos.

Os adotantes de AP da região de objeto de estudo fazem uso há, no máximo, 3 anos (Figura 2A), o que corresponde a $27 \%$ da amostra estudada. Esse período coincide com a prestação de serviço na região, que até então não existia. A aceitação da AP pelos produtores, de pequeno a médio investimento, é recente. Segundo Cirani et al. (2010), esse resultado consolida, portanto, as evidências de que, apesar de estar sendo adotada, ainda é uma tecnologia relativamente recente no país. 

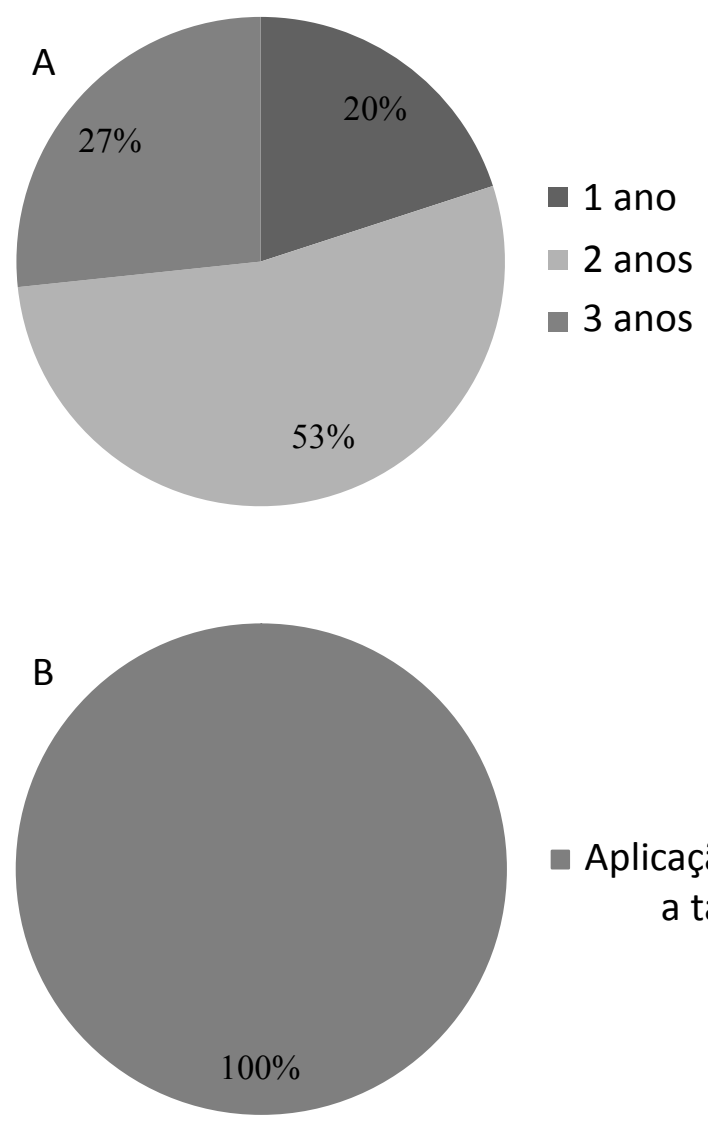

- Aplicação de insumos

a taxa variável

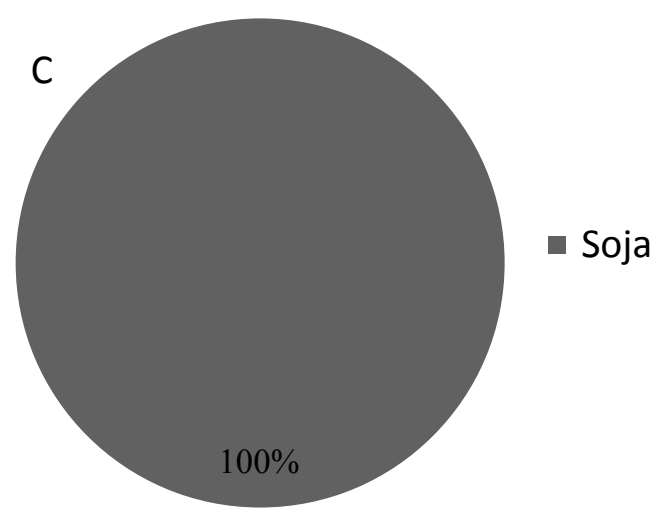

Figura 2: Tempo de utilização de ferramentas da Agricultura de Precisão (AP) (A), ferramenta utilizada (B) e cultivo agrícola que utiliza AP (C) pelos adotantes de AP

A única ferramenta utilizada na região é a aplicação de fertilizantes à taxa variável (Figura 2B) pela alta demanda dos agricultores para correção de solo da região, sendo o ponto de partida para outras ferramentas da AP. Segundo Anselmi (2012), o estágio de difusão mais adiantado dessa técnica, juntamente com a amostragem do solo georreferenciada, necessária para aplicação à taxa variada, se dá, entre outros fatores, por ser onde os produtores percebem maior possibilidade de intervenção para aumentar a produtividade e reduzir os custos, através da possível economia de fertilizantes e do pouco capital imobilizado, além da notada influência 
dos prestadores de serviços de AP na difusão destas ferramentas. A aplicação à taxa variável ainda está associada ao momento de semeadura, sendo esta realizada conforme a demanda da cultura (Silva, Moraes \& Molin, 2011).

A soja (Figura 2C), atualmente, é a principal moeda do agronegócio na região de estudo e é commodity de maior importância para o produtor rural, sendo um dos cultivos de maior retorno. Além disso, conforme discutido acima, há apenas, no máximo, 3 anos está se utilizando AP nas áreas estudadas e, por conta disso, apenas quatro produtores citaram que utilizaram a cultura do milho como forma de rotação de cultura para soja (R.17, R.21, R.22 e R.28). Resultado é reflexo do sistema de cultivo predominante no Estado do RS (Anselmi, 2012).

Através dos dados apresentados na Figura $3 \mathrm{~A}$ a respeito do aumento de produtividade com a utilização da AP, é possível observar que $40 \%$ dos produtores marcaram 4 na Escala Likert, sendo que a média ficou em 3,7. Já a respeito da melhoria da rentabilidade (Figura 3B), a maior parte dos produtores (37\%) marcaram 3 na Escala Likert, com média de 3,6. Para a obtenção de resultados concretos na $A P$, sabe-se que são necessários alguns anos para obtermos algum tipo de retorno, seja ele em rentabilidade ou em produtividade. É através do tempo que a AP demonstra sua eficiência, principalmente no que tange à recuperação/melhoria da fertilidade do solo. Os produtores rurais ainda não sabem o real custo benéfico da AP. Porém, o que a grande maioria deles procura é o aumento de produtividade (Cirani et al., 2010).

Em relação à possibilidade de identificar problemas através da utilização de ferramentas da AP (Figura 3C), 43\% dos produtores marcaram 3 na Escala Likert, apesar da média ter sido superior $(3,83)$. No que diz respeito ao custo elevado na aquisição de equipamentos e softwares (Figura 3D), 83\% dos produtores deram a nota mais elevada na Escala Likert, com a média geral em 4,83. Mesmo com um cenário em que o custo de equipamentos e softwares é elevado e a mão de obra ainda é insuficiente, os produtores rurais acreditam que é de suma importância o uso de AP na recuperação de suas áreas, pois acreditam que possam produzir mais fazendo o manejo adequado. Segundo Cirani et al. (2010) e Silva et al. (2011), o custo elevado da AP e a falta de pessoal qualificado são os maiores obstáculos.

A dificuldade de mão de obra especializada no manuseio dos equipamentos (Figura 3E) obteve média geral de 4,6 , tendo a maioria dos produtores (67\%) considerado a marcação mais elevada na Escala Likert. A utilização dessa tecnologia remete a um custo a mais na produção, pois necessita de mão de obra especializada para o uso correto e, assim, atingir os objetivos.

Quando se fala em racionalidade no uso dos insumos agrícolas através da AP, $50 \%$ dos produtores marcaram 5 na Escala Likert, porém com média geral de 4,3. (Figura 3F). O produtor rural vem utilizando essa tecnologia em sua propriedade também com a finalidade de racionalizar os insumos, assim evitando desperdícios.

Pode existir ainda limitação de tecnologias a serem superadas para o avanço de sua adoção, tais como, problemas de falta de compatibilidade dos aplicativos computacionais usados, necessidade de calibração frequente de sensores, que podem ser afetados por mudanças na topografia e pela presença de impurezas na colheita, e oferta reduzida de oficinas dotadas de equipamentos específicos e de mecânicos especializados (Cirani e Moraes, 2010). 

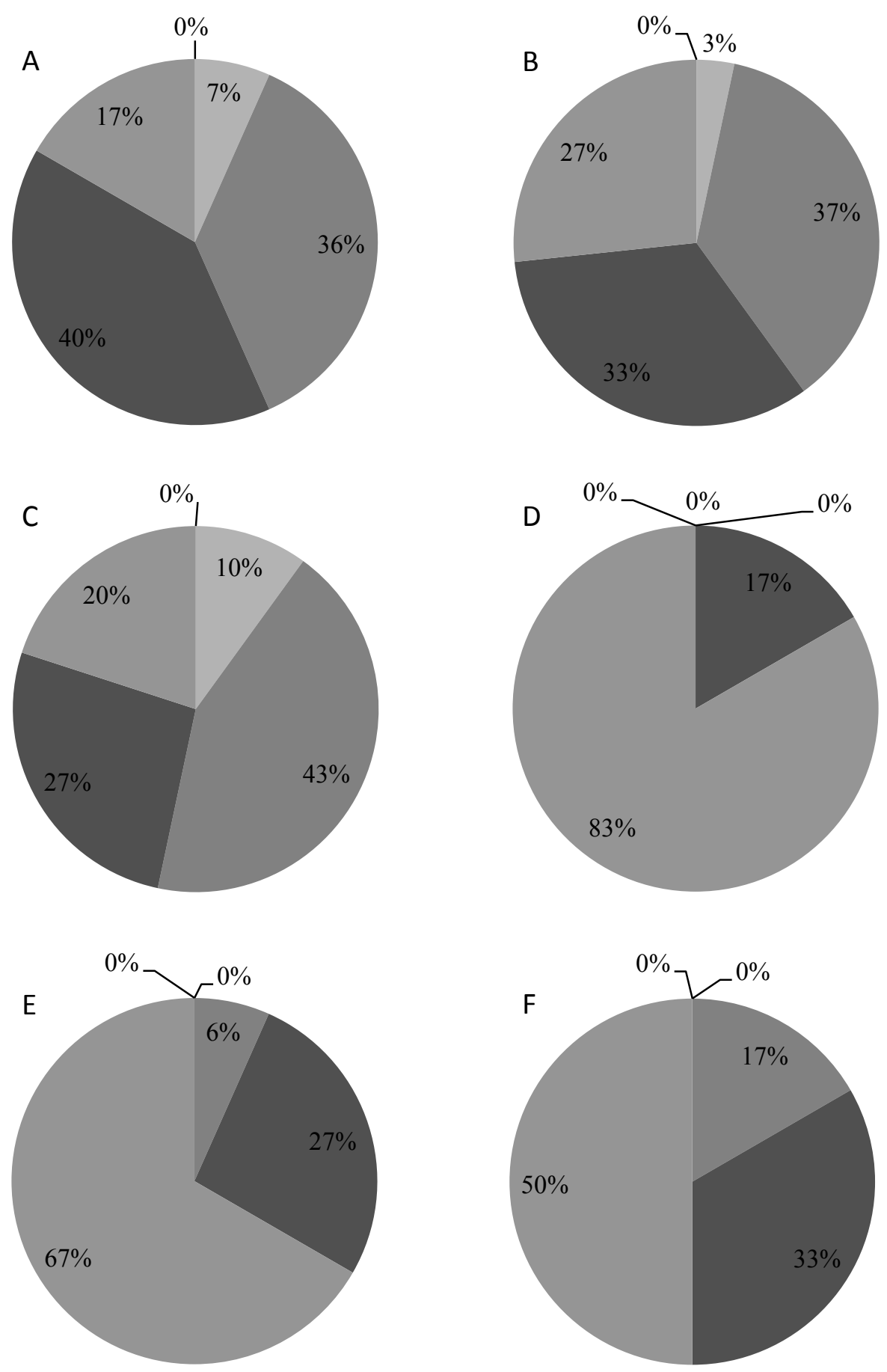

$\square 1 \square 2 \square 3 \square 4 \square 5$

Escala Likert

Figura 2: Frequência, obtida através da Escala Likert de 1 a 5 pontos, pelos adotantes de agricultura de precisão (AP). A) Conseguem-se com a AP maiores produtividades. B) A AP propicia maior rentabilidade. C) É possível identificar problemas nas áreas usando AP. D) Custos na aquisição de equipamentos e softwares são elevados. E) Dificuldade de mão de obra especializada no manuseio dos equipamentos. F) $O$ uso da AP promove maior racionalidade no uso dos insumos agrícolas. Escala Likert: 1 discordo totalmente; 5 concordo totalmente 


\section{CONCLUSÃO}

A AP ainda é recente na Região Noroeste do estado do RS. Ainda são utilizadas poucas ferramentas, restringindo-se à aplicação à taxa variável, devido ser o único serviço oferecido, e sendo utilizado principalmente na cultura da soja. É utilizada por produtores de diferentes escolaridades, idade e tamanho de área. O perfil dos produtores rurais que utilizam AP, são aqueles de pequenas e médias áreas, sendo mais viável e por ter necessidade de produzir mais em sua área que é limitada em relação ao tamanho. Em relação a idade e escolaridade, podemos observar, que quanto menor foi a idade, maior é a escolaridade, devido os filho dos produtores terem esta oportunidade de estudar primeiro para depois retornarem para suas propriedades.

\section{REFERÊNCIAS}

Adrian, A. M.; Norwood, S. H.; Maskc, P. L. (2005). Producers' perceptions and attitudes toward precision agriculture technologies. Computers and Electronics in Agriculture, 48(3), 256$271 . \quad$ Recuperado de http://www.sciencedirect.com/science/article/pii/S0168169905000852. doi: 10.1016/j.compag.2005.04.004

Anselmi, A. A. (2012). Adoção da agricultura de precisão no Rio Grande do Sul (Dissertação de Mestrado). Programa de Pós-Graduação em Agronegócios, Centro de Estudos e Pesquisas em Agronegócios, Universidade Federal do Rio Grande Do Sul - UFRGS, Porto Alegre, RS, Brasil. Recuperado de: http://www.bibliotecadigital.ufrgs.br/da.php?nrb=000826500\&loc=2012\&l=f26fc7b4ab965 $6 \mathrm{~d} 7$.

Balastreire, L. A.; Elias, A. I. e Amaral, J. R. do. (1997). Agricultura de Precisão: mapeamento da produtividade da cultura do milho. Engenharia Rural, 8(1), 97-111.

Blackmore, S.; Godwin, R.; Fountas, S. (2003). The analysis of spatial and temporal trends in yield map data over six years. Biosystems Engineering, 84(4), 455-466. Recuperado de http://www.sciencedirect.com/science/article/pii/S1537511003000382. doi: 10.1016/S1537-5110(03)00038-2

Bongiovanni, R.; Lowenberg-Deboer, J. (2004). Precision agriculture and sustainability, Precision Agriculture, 5(4), 359-387. Recuperado de https://link.springer.com/article/10.1023/B:PRAG.0000040806.39604.aa. doi: 10.1023/B:PRAG.0000040806.39604.aa

Bragagnolo, J.; Amado, T.J.C.; Nicoloso, R.S.; Santi, A.L.; Fiorin, J.E.; Tabaldi, F. (2013). Optical crop sensor for variable-rate nitrogen fertilization in corn: II - Indices of fertilizer efficiency and corn yield. Revista Brasileira de Ciência do Solo, 37(5), 1299-1309. Recuperado de http://www.scielo.br/pdf/rbcs/v37n5/19.pdf. doi: 10.1590/S0100-06832013000500019

Bramley, R. G. W. (2009). Lessons from 20 years of Precision Agriculture research, development, and adoption as a guide to its appropriate application. Crop and Pasture Science, 60(3), 197-217. Recuperado de 
https://www.researchgate.net/publication/248903745_Lessons_from_nearly_20_years_of _Precision_Agriculture_research_development_and_adoption_as_a_guide_to_its_appropr iate_application. doi: 10.1071/CP08304

Carvalho, G. R.; Botelho, C. E.; Bartholo, G. F.; Pereira, A. A.; Nogueira, A. M.; Carvalho, A. M. (2009). Comportamento de progênies F4 obtidas por cruzamentos de 'Icatu' com 'Catimor'. Ciência e Agrotecnologia, 33(1), 47-52. Recuperado de http://www.scielo.br/pdf/cagro/v33n1/v33n1a06.pdf. doi: 10.1590/S141370542009000100006

Cirani, C. B. S.; Moraes, M. A. F. D. (2010). Inovação na indústria sucroalcooleira paulista: os determinantes da adoção das tecnologias de agricultura de precisão. Revista de Economia e Sociologia Rural, 48(4), 543-565. Recuperado de http://www.scielo.br/pdf/resr/v48n4/a03v48n4.pdf. doi: 10.1590/S010320032010000400003

Cirani, C. B. S.; Moraes, M. A. F. D.; Pêssoa, L. C.; Silva, D. (2010). Uma análise de inovação a partir do estudo da adoção e uso de tecnologias de agricultura de precisão na indústria sucroalcooleira paulista. Revista de Administração e Inovação, 7(4), 186-205. Recuperado de https://www.revistas.usp.br/rai/article/view/79197/83269. doi: 10.5585/rai.2010690

Cooper, D. R. \& Schindler, P. S. (2011). Métodos de Pesquisa em Administração. (10a ed). Porto Alegre: Bookman.

Daberkow, S. G.; McBride W. D. (2003). Farm and operator characteristics affecting the awareness and adoption of precision agricultural technologies in the US. Precision Agriculture, 4(2), 163-177. Recuperado de https://link.springer.com/article/10.1023/A:1024557205871.

Demattê, J. A. M.; Demattê, J. L. I; Alves, E. R.; Barbosa, R. N.; Morelli, J. L. (2014). Precision agriculture for sugarcane management: a strategy applied for Brazilian conditions. Acta Scientiarum Agronomy, 36 (1), 111-117. Recuperado de http://periodicos.uem.br/ojs/index.php/ActaSciAgron/article/view/17664/pdf_1. doi: 10.4025/actasciagron.v36i1.17664

Ferraz G. A. E. S.; SILVA, F. M.; CARVALHO, F. M.; COSTA, P. A. N.; CARVALHO, L. C. C. (2011). Viabilidade econômica do sistema de adubação diferenciado comparado ao sistema de adubação convencional em lavoura cafeeira: um estudo de caso. Engenharia Agrícola, 31(5), 906-915. Recuperado de http://www.scielo.br/pdf/eagri/v31n5/08.pdf. doi: 10.1590/S0100-69162011000500008

Fraisse, C. (1998). Agricultura de Precisão: a tecnologia de GIS/GPS chega às fazendas. Revista Fator GIS, 21, 28-33.

Gil, A. C. (2010). Como elaborar projetos de pesquisa. (5a ed). São Paulo: Atlas.

Gimenez, L. M.; Molin, J. P. (2004). Algoritmo para redução de erros em mapas de produtividade para agricultura de precisão. Revista Brasileira de Agrocomputação, 2(1), 510, 2004. Recuperado de http://pointer.esalq.usp.br/departamentos/leb/molin/algoritmo.pdf. 
Girardello, V. C.; Amado, T.; Ertel, C.; Garlet, L. (2014b). Benefícios do tráfego controlado de máquinas. $\quad A \quad$ Granja, 34-37. Recuperado de: http://w3.ufsm.br/projetoaquarius/pdfs/artigos/_a_agranjavitorcgiradello.pdf.

Girardello, V. C.; Amado, T. J. C.; Santi, A. L.; Cherubin, M. R.; Kunz, J.; Teixeira, T. G. (2014a). Resistência à penetração, eficiência de escarificadores mecânicos e produtividade da soja em latossolo argiloso manejado sob plantio direto de longa duração. Revista Brasileira de Ciências do Solo, 38(4), 1234-1244. Recuperado de http://www.scielo.br/pdf/rbcs/v38n4/20.pdf. doi: 10.1590/S0100-06832014000400020

Goering, C. E. (1993). Recycling a concept. Agricultural Engineering, 65(6), 25.

Hair, J. F. Jr.; Babin, B., Money, A.; Samouel, P. (2005). Fundamentos de métodos de pesquisa em Administração. Porto Alegre: Bookman.

Hong, N.; Scharf, P. C.; Davis, J. G.; Kitchen, N. R.; Sudduth K. A. (2007). Economically optimal nitrogen rate reduces soil residual nitrate. Journal Environment Quality, 36(2), 354-362. Recuperado de: https://www.ncbi.nlm.nih.gov/pubmed/17255622. doi: 10.2134/jeq2006.0173

Inamasu, R. Y.; Bernardi, A. C. C.; Naime, J. M.; Queiros, L. R.; Resende, A. V.; Vilela, M. F.; Bassoi, L. H.; Perez, N. B.; Fragalle, E. P. (2011). Estratégia de implantação, gestão e funcionamento da Rede Agricultura de Precisão. In: Inamasu, R. Y.; Naime, J. M.; Resende, A. V.; Bassoi, L. H.; Bernardi, A. C. C. Agricultura de Precisão: um novo olhar. São Carlos: Embrapa Instrumentação. Recuperado de: https://www.macroprograma1.cnptia.embrapa.br/redeap2/laboratorio-nacional-deagricultura-de-precisao/livro-agricultura-de-precisao-um-novo-olhar/1.1

Mantovani, E. C.; Gomide, R. L. (2000). Agricultura de Precisão. Boletim Informativo da Sociedade Brasileira de Ciência do Solo, 25(2), 16-18.

Marsh, S. P.; Pannell, D. J.; Lindner, R. K. (2000). The impact of agricultural extension on adoption and diffusion of lupins as a new crop in Western Australia. Australian Journal of Experimental Agriculture, 40(4), 571-583. Recuperado de: https://www.researchgate.net/publication/262957227_The_impact_of_agricultural_exten sion_on_adoption_and_diffusion_of_lupins_as_a_new_crop_in_Western_Australia. doi: 10.1071/EA99080

Mc Bratney, A.; Whelan, B. e Ancev, T. (2005). Future directions of precision agriculture. Precision agriculture, 6(1), 7-23. Recuperado de https://link.springer.com/article/10.1007/s11119-005-0681-8. doi: 10.1007/s11119-0050681-8

Molin, J. P. (2002). Definição de unidades de manejo a partir de mapas de produtividade. Engenharia Agrícola, 22(1), 83-92.

Rogers, E. M. (2003). Diffusion of innovations. (5a ed.) Nova York: Free Press.

Santi, A. L.; Amado, T. J. C.; Eitelwein, M. T.; Cherubin, M. R.; Silva, R. F.; Da Ros, C. O. (2013). Definição de zonas de produtividade em áreas manejadas com Agricultura de Precisão. Revista Brasileira de Ciências Agrárias, 8(3), 510-515. Recuperado de 
http://www.agraria.pro.br/sistema/index.php?journal=agraria\&page=article\&op=view\&pa th\%5B\%5D=agraria_v8i3a2489\&path\%5B\%5D=1443. doi: 10.5039/agraria.v8i3a2489

Schlindwein, J. A.; Anghinoni, I. (2000). Variabilidade vertical de fósforo e potássio disponíveis e profundidade de amostragem do solo no sistema plantio direto. Ciência Rural, 30(4), 611617. Recuperado de http://www.scielo.br/pdf/cr/v30n4/a09v30n4.pdf. doi: $10.1590 /$ S0103-84782000000400009

Silva, F. D.; Amado, T. J. C.; Bredemeier, C.; Bremm, C.; Anghinoni, I.; Carvalho, P. C. F. (2014). Pasture grazing intensity and presence or absence of cattle dung input and its relationships to soybean nutrition and yield in integrated crop livestock systems under no-till. Agriculture, Ecosystems and Environment, 57, 84-91, 2014. Recuperado de: http://www.sciencedirect.com/science/article/pii/S1161030113001500. doi: 10.1016/j.eja.2013.10.009

Silva, C. B.; Moraes, M. A. F. D.; Molin, J. P. (2011). Adoption and use of precision agriculture technologies in the sugarcane industry of São Paulo state, Brazil. Precision Agriculture, 12(1), 67-81. Recuperado de: https://link.springer.com/article/10.1007/s11119-009-91558. doi: 10.1007/s11119-009-9155-8

Teixeira, G. (2005). Conheça o que são Escalas de Medida. Recuperado de: http://www.serprofessoruniversitario.pro.br/ler.php?modulo=21\&texto=1304.

Toledo, L. G.; Nicolella, G. (2002). Índice de qualidade de água em microbacia sob uso agrícola e urbano. Scientia Agricola, 59 (1), 181-186, 2002. Recuperado de: http://www.scielo.br/pdf/sa/v59n1/8092.pdf. doi: 10.1590/S0103-90162002000100026

Vergara, S. C. (2011). Projeto e Relatório de Pesquisa em Administração. São Paulo: Atlas. 